\title{
Transient and steady-state rotation centre of vehicle dynamics
}

\author{
Hormoz Marzbani and D.Q. Vo \\ School of Engineering, \\ RMIT University, \\ Melbourne, VIC, Australia \\ Email: hormoz.marzbani@rmit.edu.au \\ Email: dai.voquoc@rmit.edu.au
}

\author{
Ali Khazaei* \\ ME Department, \\ Southern Polytechnic College of \\ Engineering \& Engineering Technology, \\ Kennesaw State University (KSU), \\ Kennesaw, GA, USA \\ Email: akhazaei@kennesaw.edu \\ *Corresponding author
}

\section{Mohammad Fard and Reza N. Jazar}

School of Engineering,

RMIT University,

Melbourne, VIC, Australia

Email: mohammad.fard@rmit.edu.au

Email: reza.jazar@rmit.edu.au

\begin{abstract}
The effect of acceleration - variable forward velocity, have been investigated on the steady-state and transient responses of turning vehicles and the resulted path of motion of the vehicle. Comparing the two mentioned responses of the vehicle could be used to prove that there is a negligible difference between the steady-state and transient centre of rotation of the vehicle in engineering applications. Dynamics of a vehicle with a constant steer angle and variable forward velocity have been analysed and presented as the proof. It has been shown that it is possible to predict the dynamics of vehicles using their steady state responses within acceptable engineering approximations. More specifically, we determine the dynamic rotation centre of vehicles and compare them with steady-state values. The result would be essential to design autodriver algorithm for autonomous vehicles.
\end{abstract}

Keywords: vehicle dynamics; steady-state vehicle behaviour; rotation centre of vehicles; variable forward velocity. 
Reference to this paper should be made as follows: Marzbani, H., Vo, D.Q., Khazaei, A., Fard, M. and Jazar, R.N. (2017) 'Transient and steady-state rotation centre of vehicle dynamics', Int. J. Nonlinear Dynamics and Control, Vol. 1, No. 1, pp.97-113.

Biographical notes: Hormoz Marzbani is a Lecturer in the School of Engineering, RMIT University. He gained industrial experience when working as a Product Engineer in automotive industry and as a Design Engineer in the materials handling industry. He has published more than 20 papers, book chapters as author and co-author. His areas of interest include: dynamics and vibration, vehicle noise and vibration, and autonomous vehicles.

D.Q. Vo is currently working toward his $\mathrm{PhD}$ in Automotive Engineering at School of Engineering, RMIT University, Australia. His research interests include kinematics, dynamics of vehicle systems; variable caster steering; dynamics, ride, handling, and stability of vehicles. He has published several peer-reviewed journal papers and book chapters.

Ali Khazaei has long industrial experience (1983-2008) (e.g., eight years with Newcomb \& Boyd, a consulting firm in USA). He has 12 years experience as a full-time faculty (1988-2000) and eight years (Fall 2008-Present) at SPSU (which merged with KSU). He has taught 15 different courses in three different fields ('solid', 'fluid' and 'heat') before joining SPSU (2008) and has taught ten different courses (all major courses in energy program). He has developed five new courses. He received TADL Certificate, Developed and Taught online courses at SPSU (which merged with KSU). He initiated new energy program entitled 'Energy Concentration in MET Department'. He has published 13 (peer reviewed) articles ( 2 journal articles +11 conference articles).

Mohammad Fard leads the Noise and Vibration Laboratory in the School of Engineering, RMIT University. He gained industrial experience when working at Nissan Motor Co, Japan (2003-2009). He has published more than 75 papers, book chapters as author and co-author. His areas of interest include: biomechanics, vehicle body CAE concept modelling, vehicle noise and vibration, characterisation of sound absorption of porous material, signal processing, and frequency domain identification method.

Reza N. Jazar is the Discipline Head of Mechanical and Automotive Engineering, School of Engineering, RMIT University. He has several years of practical industrial experience mostly in vehicle manufacturing and automotive industries all around the world. He is also an active member of many journals, publishers, conferences, and organisations as an editor, reviewer, or organizer. $\mathrm{He}$ is the Editor in Chief of Journal of Nonlinear Engineering. He has authored or co-authored over 200 publications including books, journal articles, and conference papers. His research interests include: dynamics, handling, ride, and stability of vehicles, autonomous vehicles and smart/safe vehicles.

\section{Introduction}

The equations of motion of the planar bicycle car model, expressed in the principal body coordinate frame $B$, is governed by the following set of nonlinear coupled ordinary differential equations. In the equations, the steering angle $\delta$ acts as the input and, forward 
velocity of the centre of the mass of the vehicle $v_{x}$, lateral velocity $v_{y}$, and yaw rate $r$, are the outputs (Jazar, 2013).

$$
\begin{aligned}
& \dot{v}_{x}=\frac{1}{m} F_{x}+r v_{y} \\
& \dot{v}_{y}=\frac{C_{\beta}}{m v_{x}} v_{y}-\left(v_{x}-\frac{C_{r}}{m}\right) r+\frac{C_{\delta}}{m} \delta(t) \\
& \dot{r}=\frac{D_{\beta}}{I_{z} v_{x}} v_{y}+\frac{D_{r}}{I_{z}} r+\frac{D_{\delta}}{I_{z}} \delta(t)
\end{aligned}
$$

where

$$
\begin{aligned}
& C_{r}=\frac{\partial F_{y}}{\partial r}=-\frac{a_{1}}{v_{x}} C_{\alpha f}+\frac{a_{2}}{v_{x}} C_{\alpha r} \\
& C_{\beta}=\frac{\partial F_{y}}{\partial \beta}=-C_{\alpha f}-C_{\alpha r} \\
& C_{\delta}=\frac{\partial F_{y}}{\partial \delta}=C_{\alpha f} \\
& D_{r}=\frac{\partial M_{z}}{\partial r}=-\frac{a_{1}^{2}}{v_{x}} C_{\alpha f}-\frac{a_{2}^{2}}{v_{x}} C_{\alpha r} \\
& D_{\beta}=\frac{\partial M_{z}}{\partial \beta}=a_{2} C_{\alpha r}-a_{1} C_{\alpha f} \\
& D_{\delta}=\frac{\partial M_{z}}{\partial \delta}=a_{1} C_{\alpha f} \\
& \beta=\frac{v_{y}}{v_{x}}
\end{aligned}
$$

The orthogonal body coordinate frame $B$ is fixed to the vehicle at its mass centre $C$ and is set such that the $x$-axis is longitudinal, $y$-axis is lateral, and $z$-axis is vertical upwards. The body coordinate and kinematics of the bicycle car model in a forward motion on a positive turn are illustrated in Figure 1.

The coefficients $C_{r}, C_{\beta}, C_{\delta}, D_{r}, D_{\beta}, D_{\delta}$ in the equations of motion are slopes of the curves for lateral force $F_{y}$ and yaw moment $M_{z}$ as a function of $r, \beta$, and $\delta$ respectively. The coefficients $C_{r}$ and $D_{r}$ are functions of $v_{x}$, and the coefficients $C_{\beta}, C_{\delta}, D_{\beta}, D_{\delta}$ are constant for a given vehicle. In this case it is been assumed that the vehicle is moving with a constant steer angle equal to:

$$
\delta(t)=0.1 \mathrm{rad}=5.37 \mathrm{deg}
$$

The forward velocity of the vehicle changes with time according to the following function: 


$$
v_{x}=\frac{20}{t_{0}} t H\left(t_{0}-t\right)+20 H\left(t-t_{0}\right) \mathrm{m} / \mathrm{s}
$$

where $v_{x}$ changes from $v_{x}=0 \mathrm{~m} / \mathrm{s}$ at $t=0$ to the maximum speed $v_{x}=20 \mathrm{~m} / \mathrm{s}$ at $t=t_{0}$, and remains constant afterwards. $H\left(t-t_{0}\right)$ is the Heaviside function:

$$
H\left(t-t_{0}\right)= \begin{cases}0 & t<t_{0} \\ 1 & t>t_{0}\end{cases}
$$

Figure 1 Kinematics of a moving vehicle at steady-state conditions

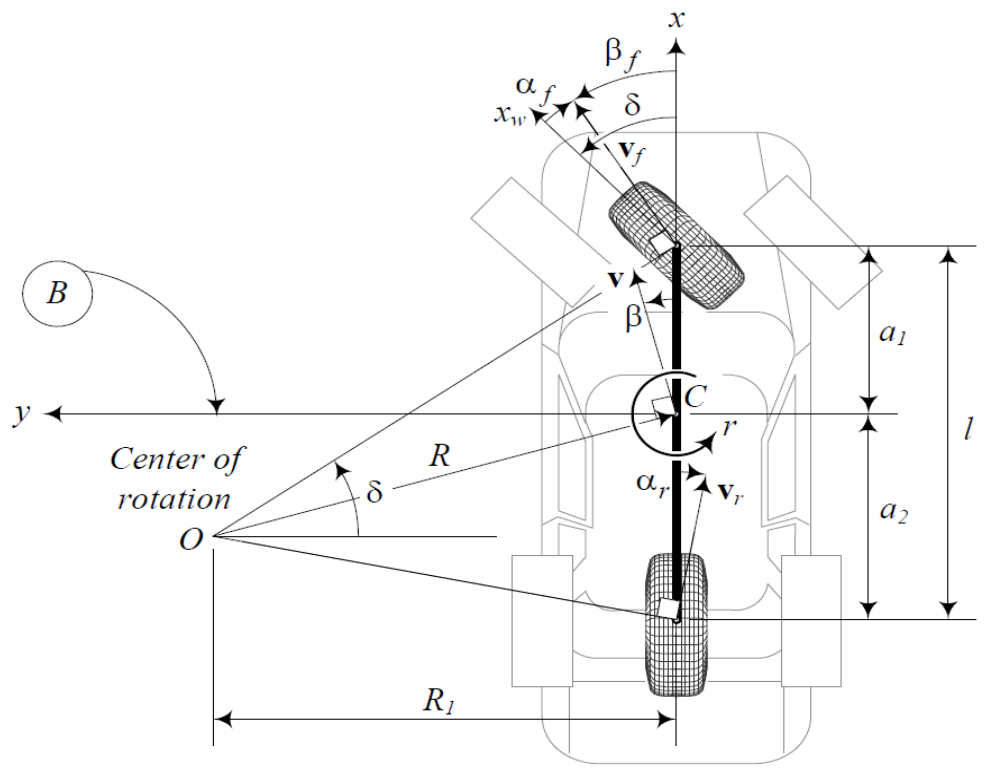

The equations of motion of a two-wheel rigid vehicle with no roll are as the following:

$$
\begin{aligned}
& \dot{v}_{x}=\frac{1}{m} F_{x}+r v_{y} \\
& {\left[\begin{array}{l}
\dot{v}_{y} \\
\dot{r}
\end{array}\right]=\left[\begin{array}{ll}
\frac{C_{\beta}}{m v_{x}} & \frac{C_{r}}{m}-v_{x} \\
\frac{D_{\beta}}{I_{z} v_{x}} & \frac{D_{r}}{I_{z}}
\end{array}\right]\left[\begin{array}{l}
v_{y} \\
r
\end{array}\right]+\left[\begin{array}{c}
\frac{C_{\beta}}{m} \\
\frac{D_{\delta}}{I_{z}}
\end{array}\right] \delta(t)}
\end{aligned}
$$

When $v_{x}$ is known, either is constant or varying with time, equation (15) becomes independent from (14). The set of equation (15) can then be written in the form

$$
\dot{\mathbf{q}}=[A] \mathbf{q}+\mathbf{u}
$$

in which $[A]$ is a constant coefficient matrix, $\mathbf{q}$ is the vector of control variables, and $\mathbf{u}$ is the vector of inputs. 


$$
\begin{aligned}
{[A] } & =\left[\begin{array}{ll}
\frac{C_{\beta}}{m v_{x}} & \frac{C_{r}}{m}-v_{x} \\
\frac{D_{\beta}}{I_{z} v_{x}} & \frac{D_{r}}{I_{z}}
\end{array}\right] \\
& =\left[\begin{array}{ll}
-\frac{C_{\alpha f}+C_{\alpha r}}{m v_{x}} & \frac{-a_{1} C_{\alpha f}+a_{2} C_{\alpha r}}{m v_{x}}-v_{x} \\
-\frac{a_{1} C_{\alpha f}-a_{2} C_{\alpha r}}{I_{z} v_{x}} & -\frac{a_{1}^{2} C_{\alpha f}+a_{2}^{2} C_{\alpha r}}{I_{z} v_{x}}
\end{array}\right] \\
\mathbf{q} & =\left[\begin{array}{l}
v_{y} \\
r
\end{array}\right] \delta(t)=\left[\begin{array}{l}
\frac{C_{\beta}}{m} \\
\frac{D_{\delta}}{I_{z}}
\end{array}\right] \delta(t) \\
\mathbf{u} & =\left[\begin{array}{l}
\frac{C_{\alpha}}{a_{1} C_{\alpha f}} \\
I_{z}
\end{array}\right] \delta(
\end{aligned}
$$

The following characteristics for a sample car will be used to develop the equation of motion.

$$
\begin{array}{ll}
C_{\alpha f}=50,000 \mathrm{~N} / \mathrm{rad} & C_{\alpha r}=50,000 \mathrm{~N} / \mathrm{rad} \\
m=1,000 \mathrm{~kg} & I_{z}=1,650 \mathrm{kgm}^{2} \\
a_{1}=1.0 \mathrm{~m} & a_{2}=1.5 \mathrm{~m}
\end{array}
$$

In the following sections, we calculate the transient response of an understeer passenger car that is moving with a constant steering angle equal to equation (11) and a variable forward velocity which changes with time according to equation (12). The transition behaviour of the car will be determined using steady-state responses to examine the proximity of the two analysis. It will be shown that steady-state response equations are good enough to predict the transition behaviour of the car.

The results of this investigation are expected to be useful in speeding up the online calculation of autodriver algorithm (Jazar, 2010), as well as obtaining data to determine the steady-state behaviour of vehicles (Tahami et al., 2003).

\section{Steady-state responses}

When the vehicle is turning at a steady-state condition, its behaviour is governed by the following equations:

$$
\begin{aligned}
& F_{x}=-\frac{m}{R} v_{x} v_{y} \\
& C_{\beta} \beta+\left(C_{r} v_{x}-m v_{x}^{2}\right) \frac{1}{R}=-C_{\delta} \delta
\end{aligned}
$$




$$
D_{\beta} \beta+D_{r} v_{x} \frac{1}{R}=-D_{\delta} \delta
$$

from which, we can define a set of steady-state responses (Jazar, 2013):

1 curvature response, $S_{\kappa}$

$$
S_{\kappa}=\frac{\kappa}{\delta}=\frac{1}{R \delta}=\frac{C_{\delta} D_{\beta}-C_{\beta} D \delta}{v_{x}\left(D_{r} C_{\beta}-C_{r} D_{\beta}+m v_{x} D_{\beta}\right)}
$$

2 sideslip response, $S_{\beta}$

$$
S_{\beta}=\frac{\beta}{\delta}=\frac{D \delta\left(C_{r}-m v_{x}\right)-D_{r} C_{\delta}}{D_{r} C_{\beta}-C_{r} D_{\beta}+m v_{x} D_{\beta}}
$$

3 yaw rate response, $S_{r}$

$$
S_{r}=\frac{r}{\delta}=\frac{\kappa}{\delta} v_{x}=S_{\kappa} v_{x}=\frac{C_{\delta} D_{\beta}-C_{\beta} D \delta}{D_{r} C_{\beta}-C_{r} D_{\beta}+m v_{x} D_{\beta}}
$$

4 centripetal acceleration response, $S_{a}$

$$
S_{a}=\frac{v_{x}^{2} / R}{\delta}=\frac{\kappa}{\delta} v_{x}^{2}=S_{\kappa} v_{x}^{2}=\frac{\left(C_{\delta} D_{\beta}-C_{\beta} D \delta\right) v_{x}}{D_{r} C_{\beta}-C_{r} D_{\beta}+m v_{x} D_{\beta}}
$$

5 lateral velocity response, $S_{y}$

$$
S_{y}=\frac{v_{y}}{\delta}=S_{\beta} v_{x}=\frac{D \delta\left(C_{r}-m v_{x}\right)-D_{r} C_{\delta}}{D_{r} C_{\beta}-C_{r} D_{\beta}+m v_{x} D_{\beta} v_{x}} .
$$

Figure 2 illustrates steady-state responses for the sample car mentioned above.

Figure 2 Steady-state responses of a sample vehicle, as functions of forward velocity $v_{x}$ (see online version for colours)

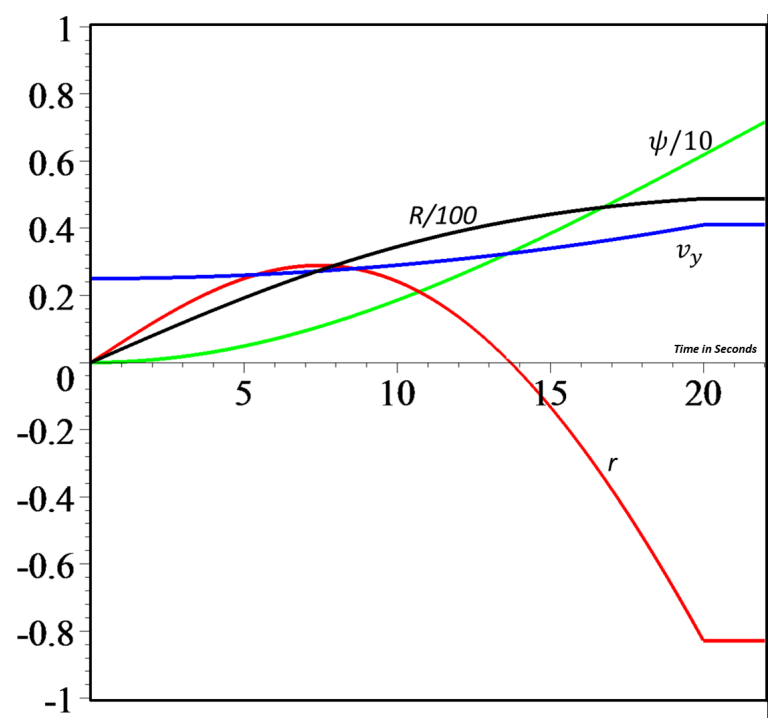


The vehicle is understeer because of a positive stability parameter $K$ (Jazar, 2013)

$$
K=\frac{m}{l^{2}}\left(\frac{a_{2}}{C_{\alpha f}}-\frac{a_{1}}{C_{\alpha r}}\right)=0.0016>0
$$

And the steady-state responses for the case of variable speed will be:

1 curvature response, $S_{\kappa}$

$$
S_{\kappa}=\frac{0.625 \times 10^{10}}{Z\left(\frac{0.15625 \times 10^{11}}{Z}+0.25 \times 10^{8} t H(-t+20)+0.5 \times 10^{9} H(t-20)\right)}
$$

2 sideslip response, $S_{\beta}$

$$
S_{\beta}=\frac{\frac{0.9375 \times 10^{10}}{Z}-0.5 \times 10^{8} t H(-t+20)-0.1 \times 10^{10} H(t-10)}{\frac{0.15625 \times 10^{11}}{Z}+0.25 \times 10^{8} t H(-t+20)+0.5 \times 10^{9} H(t-10)}
$$

3 yaw rate response, $S_{r}$

$$
S_{r}=\frac{0.625 \times 10^{10}}{\frac{0.15625 \times 10^{11}}{Z}+0.25 \times 10^{8} t H(-t+20)+0.5 \times 10^{9} H(t-20)}
$$

4 centripetal acceleration response, $S_{a}$

$$
S_{a}=\frac{0.625 \times 10^{10}(Z)}{\frac{0.15625 \times 10^{11}}{Z}+0.25 \times 10^{8} t H(-t+20)+0.5 \times 10^{9} H(t-20)}
$$

5 lateral velocity response, $S_{y}$

$$
S_{y}=\frac{\left(\frac{0.9375 \times 10^{10}}{Z}-0.5 \times 10^{8} t H(-t+20)-0.1 \times 10^{10} H(t-20)\right) Z}{\frac{0.15625 \times 10^{11}}{Z}+0.25 \times 10^{8} t H(-t+20)+0.5 \times 10^{9} H(t-20)}
$$

where $Z=t H(-t+20)+20 H(t-20)$.

Employing the steady-state responses, we can determine the variation of the kinematics of motion as functions of time $t$.

\section{Steady-state rotation centre and path of motion}

Having the steady-state responses $S_{k}=1 / R / \delta$ and $S_{\beta}=\beta / \delta$, we are able to determine the steady-state position of the rotation centre $\left(x_{O}, y_{O}\right)$ of a vehicle in the vehicle body coordinate frame as is illustrated in Figure 3. 


$$
\begin{aligned}
& x_{O}=-R \sin \beta=-\frac{1}{S_{k} \delta} \sin \left(S_{\beta} \delta\right) \\
& y_{O}=R \cos \beta=\frac{1}{S_{k} \delta} \cos \left(S_{\beta} \delta\right)
\end{aligned}
$$

Figure 3 The coordiantes $\left(x_{O_{\text {steady }}}, y_{O_{\text {steady }}}\right)$ of the steady-state rotation centre of the vehicle in the body frame for $0 \leq t \leq 20 \mathrm{~s}$

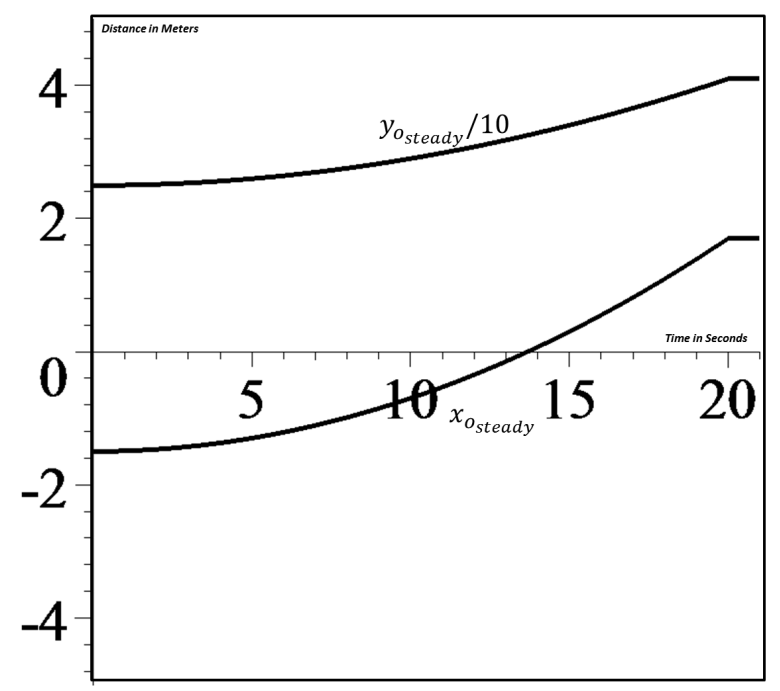

At steady-state conditions the radius of rotation $R$ can be found from the curvature response $S_{\kappa}$, equation (30), and the vehicle sideslip angle $\beta$ from the sideslip response $S_{\beta}$ [equation (31)].

$$
\begin{aligned}
& R=\frac{1}{\delta S_{\kappa}}=\frac{v_{x}\left(D_{r} C_{\beta}-C_{r} D_{\beta}+m v_{x} D_{\beta}\right)}{\left(C_{\delta} D_{\beta}-C_{\beta} D_{\delta}\right) \delta} \\
& \beta=\delta S_{\beta}=\frac{D_{\delta}\left(C_{r}-m v_{x}\right)-D_{r} C_{\delta}}{D_{r} C_{\beta}-C_{r} D_{\beta}+m v_{x} D_{\beta}} \delta
\end{aligned}
$$

Therefore, the steady-state position of the dynamic centre of rotation $O$ in the body coordinate frame $B$, about which the vehicle will actually turn, is at

$$
\begin{aligned}
& x_{O}=-\frac{v_{x}\left(D_{r} C_{\beta}-C_{r} D_{\beta}+m v_{x} D_{\beta}\right)}{\left(C_{\delta} D_{\beta}-C_{\beta} D \delta\right) \delta} \sin \left(\frac{D \delta\left(C_{r}-m v_{x}\right)-D_{r} C_{\delta}}{D_{r} C_{\beta}-C_{r} D_{\beta}+m v_{x} D_{\beta}} \delta\right) \\
& y_{O}=\frac{v_{x}\left(D_{r} C_{\beta}-C_{r} D_{\beta}+m v_{x} D_{\beta}\right)}{\left(C_{\delta} D_{\beta}-C_{\beta} D_{\delta}\right) \delta} \cos \left(\frac{D_{\delta}\left(C_{r}-m v_{x}\right)-D_{r} C_{\delta}}{D_{r} C_{\beta}-C_{r} D_{\beta}+m v_{x} D_{\beta}} \delta\right)
\end{aligned}
$$

Figure 4 illustrates the coordinates $x_{O_{\text {steady }}}$ and $y_{O_{\text {steady }}}$ of the vehicle for $0 \leq t \leq 20$ after substituting equation (12) in to equations (39) and (40) At a constant steer angle, the 
dynamic rotation centre of the understeer vehicle moves away and forward by increasing the forward velocity. The rate of the displacement of the rotation centre increases by speed. Figure 4 depicts how location of the centre of rotation with respect to the body frame of the vehicle changes with speed. It indicates the critical speed at which the velocity vector of the vehicle at its mass centre is in the $x$ direction only. At the critical speed, we have $\beta=0$ and the dynamics centre of rotation is located on the $y$-axis.

Figure 4 The location of the steady-state rotation centre in the body frame for $0 \leq v_{x} \leq 20$

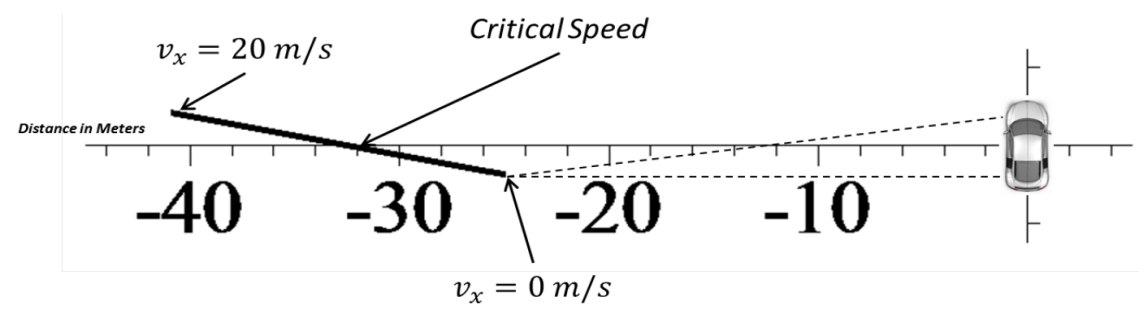

The global frame $G$ is fixed to the ground, and $B$ coincides $G$ at the beginning of manoeuvres. The $B$-frame moves with the vehicle while the axes $z$ and $Z$ are always parallel. Therefore, the velocity vector of the vehicle in the global frame is

$$
\begin{aligned}
{ }^{G} \mathbf{v} & ={ }^{G} R_{B}{ }^{B} \mathbf{v}=\left[\begin{array}{rr}
\cos \psi & -\sin \psi \\
\sin \psi & \cos \psi
\end{array}\right]\left[\begin{array}{l}
v_{x} \\
v_{y}
\end{array}\right] \\
& =\left[\begin{array}{l}
v_{x} \cos \psi-v_{y} \sin \psi \\
v_{y} \cos \psi+v_{x} \sin \psi
\end{array}\right]=\left[\begin{array}{l}
v_{X} \\
v_{Y}
\end{array}\right]
\end{aligned}
$$

where ${ }^{G} R_{B}$ is the transformation between $B$ and $G$ and the velocity vector of the vehicle in the body frame is

$$
{ }^{B} \mathbf{v}=\left[\begin{array}{l}
v_{x} \\
v_{y}
\end{array}\right]
$$

Therefore, the global coordinates of the mass centre of the vehicle would be

$$
\begin{aligned}
& X=\int_{0}^{t} v_{X} d t==\int_{0}^{t}\left(v_{x} \cos \psi-v_{y} \sin \psi\right) d t \\
& Y=\int_{0}^{t} v_{Y} d t=\int_{0}^{t}\left(v_{y} \cos \psi+v_{x} \sin \psi\right) d t
\end{aligned}
$$

When the steer angle is kept constant, and after reaching the top speed of $20 \mathrm{~m} / \mathrm{s}$ the vehicle will eventually be turning on a constant circular path. The position of the steady-state rotation centre of the vehicle in the $B$-frame is at

$$
\begin{aligned}
& x_{\text {Osteady }}=-R \sin \beta=1.6994 \mathrm{~m} \\
& y_{\text {Osteady }}=R \cos \beta=40.965 \mathrm{~m}
\end{aligned}
$$

because 


$$
\begin{aligned}
& \lim _{t \rightarrow \infty} R=41 \mathrm{~m} \\
& \lim _{t \rightarrow \infty} \beta=-0.04146 \mathrm{rad}
\end{aligned}
$$

The global coordinates of the steady-state rotation centre is

$$
\left[\begin{array}{c}
X_{O_{\text {steady }}} \\
Y_{O_{\text {steady }}}
\end{array}\right]={ }_{G}^{G} \mathbf{r}_{B}+{ }^{G} R_{B}\left[\begin{array}{l}
x_{O_{\text {steady }}} \\
y_{O_{\text {steady }}}
\end{array}\right]=\left[\begin{array}{l}
-5.8 \mathrm{~m} \\
-23.9 \mathrm{~m}
\end{array}\right]
$$

where ${ }_{O}^{G} \mathbf{r}_{O}$ is the $G$-expression of the position vector of the origin of the $B$-frame with respect to the origin of the $G$-frame at any point on the steady-state conditions (Jazar, 2011). In this example, we used the calculated data at $t=2 \mathrm{~s}$ when we have

$$
\begin{aligned}
& {\left[\begin{array}{l}
x_{O_{\text {steady }}} \\
y_{O_{\text {steady }}}
\end{array}\right]=\left[\begin{array}{l}
1.6994 \mathrm{~m} \\
40.965 \mathrm{~m}
\end{array}\right]} \\
& \psi=3.84 \mathrm{rad} \\
& { }_{O}^{G} \mathbf{r}_{O}=\left[\begin{array}{l}
-27.345 \mathrm{~m} \\
-50.182 \mathrm{~m}
\end{array}\right]
\end{aligned}
$$

Figure 5 The steady-state path of motion of the vehicle and its rotation centre (see online version for colours)

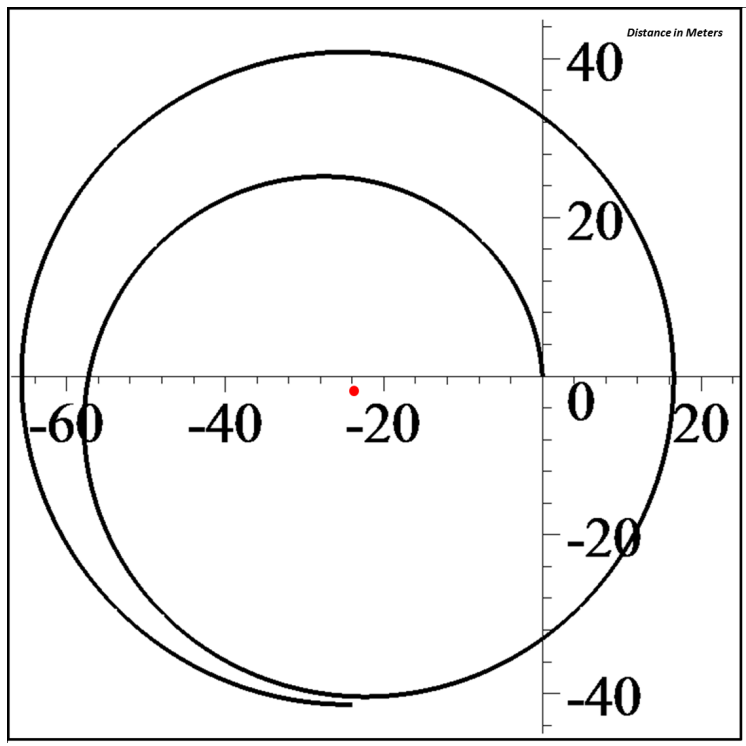

Figure 5 illustrates the instantaneous path of motion of the vehicle in global frame and its final steady-state rotation centre. The magnification of the transient stage depicts how the vehicle approaches its steady state circular path. 


\section{Dynamic solution rotation centre and path of motion}

In order to be able to compare the dynamic solution results which will be called the 'exact solution' from now on with the steady-state solutions called 'approximate solution' let's solve equations (15), (16) and (19). The equations of motion will turn to the following after substituting all the variables and nominal values from the sample car:

$$
\begin{aligned}
& \dot{v}_{y}(t)=\left(\frac{25}{t H(-t+20)+20 H(t-20)}-t H(-t+20)-20 H(t-20)\right) r(t)-5 \\
& \dot{r}(t)=\frac{15.1515 v_{y}(t)}{t H(-t+20)+20 H(t-20)}+\frac{98.4848 r(t)}{t H(-t+20)+20 H(t-20)}-3.03
\end{aligned}
$$

These are exactly equal to the same values for the approximate solutions in equations (47) and (48). This means that the path of motion for both solutions is exactly the same after they get to the steady-state condition. So, every deviation in the final path of motion of the two solutions has happened during the transition time.

The centre of rotation of the vehicle in the body frame $\left(X_{o}, Y_{o}\right)$ are found as the following:

$$
\left[\begin{array}{l}
x_{O} \\
y_{O}
\end{array}\right]=\left[\begin{array}{l}
1.6994 \mathrm{~m} \\
40.965 \mathrm{~m}
\end{array}\right]
$$

which are again the same, which indicates that the path of motion taken by the vehicle in both situations will look the same from the eye of the passengers of the car.

Figure 6 The coordinate $\left(x_{O}, y_{O}\right)$ of the steady-state rotation centre of the vehicle in the body frame for $0 \leq t \leq 20 \mathrm{~m}$

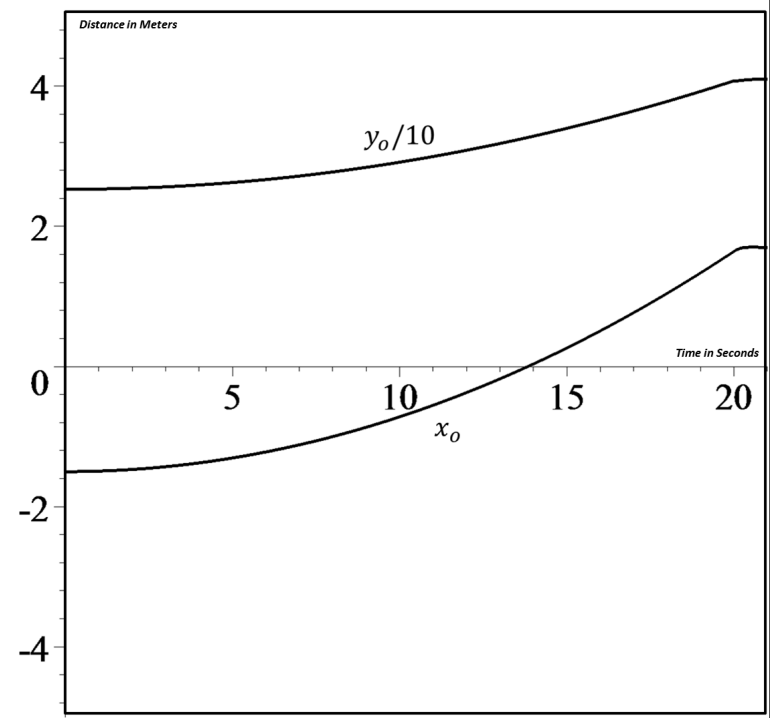


Figure 7 The location of the dynamic rotation centre in the body frame for $0 \leq v_{x} \leq 20 \mathrm{~m} / \mathrm{s}$

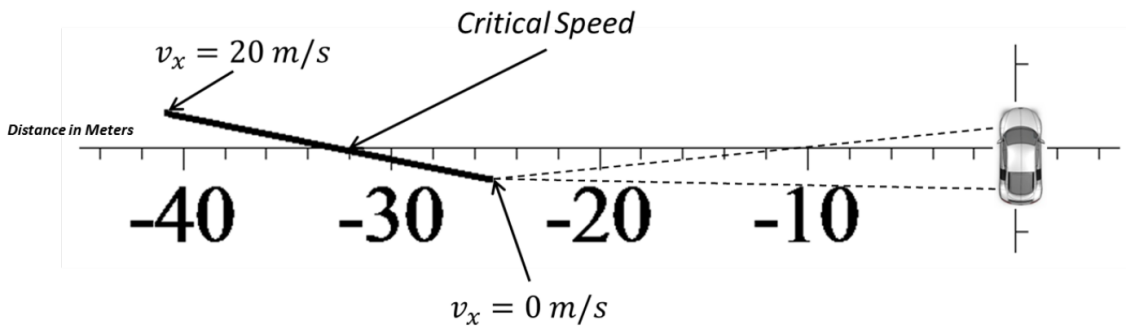

But in order to find the centre of rotation in the global frame we will need to repeat the equations (43) and (44). Following the same steps as before we will get:

$$
\begin{aligned}
& \psi=3.82 \mathrm{rad} \\
& { }_{O}^{G} \mathbf{r}_{O}=\left[\begin{array}{l}
-27.345 \mathrm{~m} \\
-50.182 \mathrm{~m}
\end{array}\right]
\end{aligned}
$$

We will get,

$$
\left[\begin{array}{c}
X_{O} \\
Y_{O}
\end{array}\right]={ }_{O}^{G} \mathbf{r}_{O}+{ }^{G} R_{B}\left[\begin{array}{c}
x_{O} \\
y_{O}
\end{array}\right]=\left[\begin{array}{l}
-5.61 \mathrm{~m} \\
-24.22 \mathrm{~m}
\end{array}\right]
$$

Figure 8 The dynamics response (exact) path of motion of the vehicle and its rotation centre (see online version for colours)

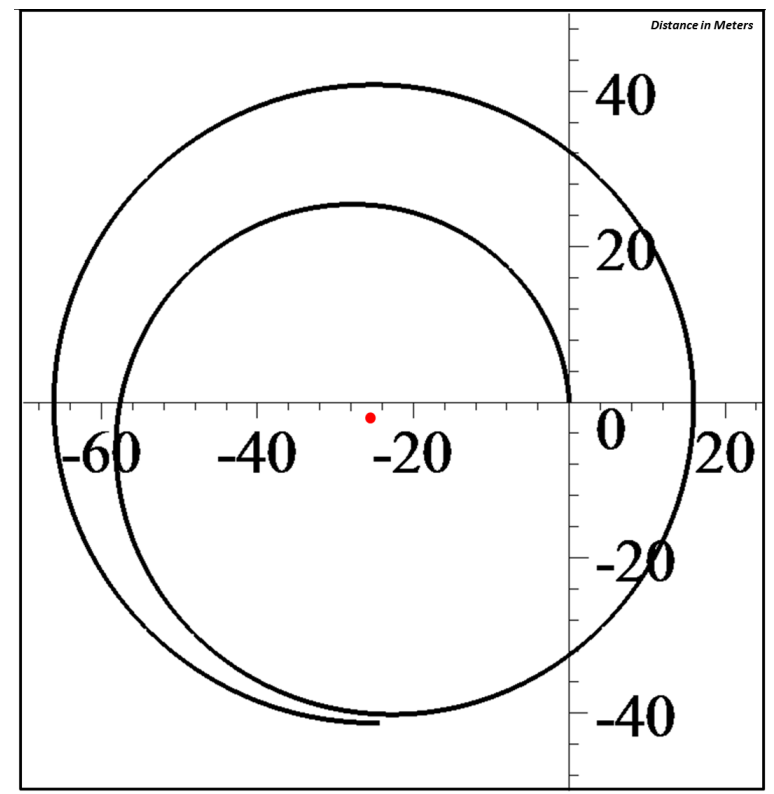




\section{Conclusions}

The steady-state responses of a vehicle which starts moving with a constant steering angle, while the forward velocity is changing with time has been calculated for a sample car. The same sample has been used to solve the problem with the same condition dynamically. The final path of motion has been calculated for both of the above conditions. By comparing the exact and approximate paths of motion we will be able to decide whether the approximate method is accurate enough for engineering purposes. The reason behind all these is to make the calculation expenses for autonomous algorithms for autodriver shorter and less complicated. There will be two methods for comparing the two resulted paths of motion. The first method is to compare the position of the vehicle at any moment of time on the two paths and check the distance between the positions of the two. The difference resulted in this case will include the transition time lag for the exact path which will cause the vehicle to fall behind. The reason is that for the approximate method no time is taken by the system to achieve the steady-state condition. For this case the values used for calculating the global position of the centre of rotation can be used for calculating the global position of the centre of rotation can be used from equations (52) and (59). These are the actual positions of the vehicle on the paths of motion at time $t=14.985 \mathrm{~s}$.

These values indicate that the two vehicles are just $0.624 \mathrm{~m}$ apart in the $x$ direction and $0.668 \mathrm{~m}$ apart in the $y$ direction. The total distance of the two paths of motion at this moment of time is only $0.91 \mathrm{~m}$ which is only $2.2 \%$ of the radius of rotation. Figures 9 and 10 indicate the deviation in the $x$ and $y$ direction during the time of motion which makes it possible to find out the maximum deviation of the two resulted paths.

Figure 9 Deviation in the $x$ direction - time lag included

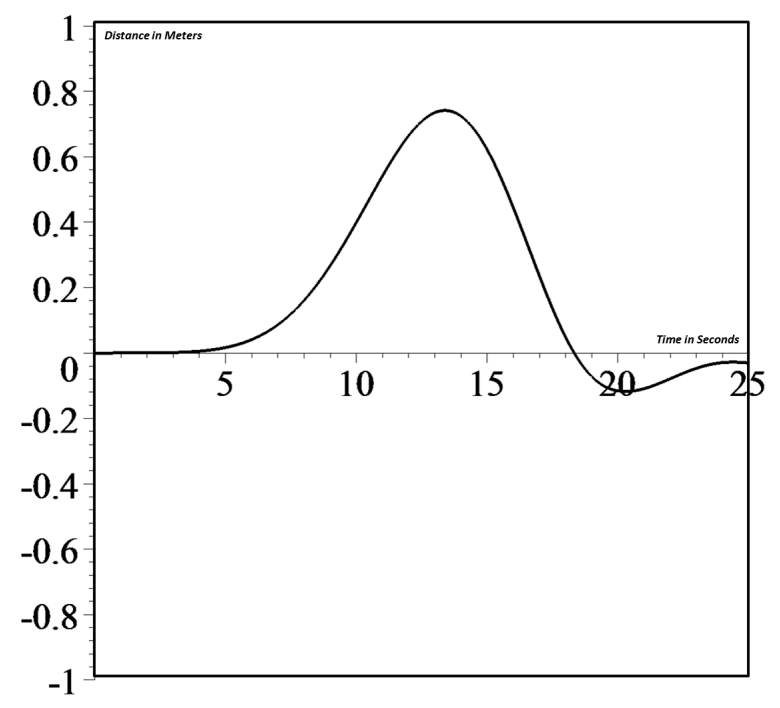


Figure 10 Deviation in the $y$ direction - time lag included

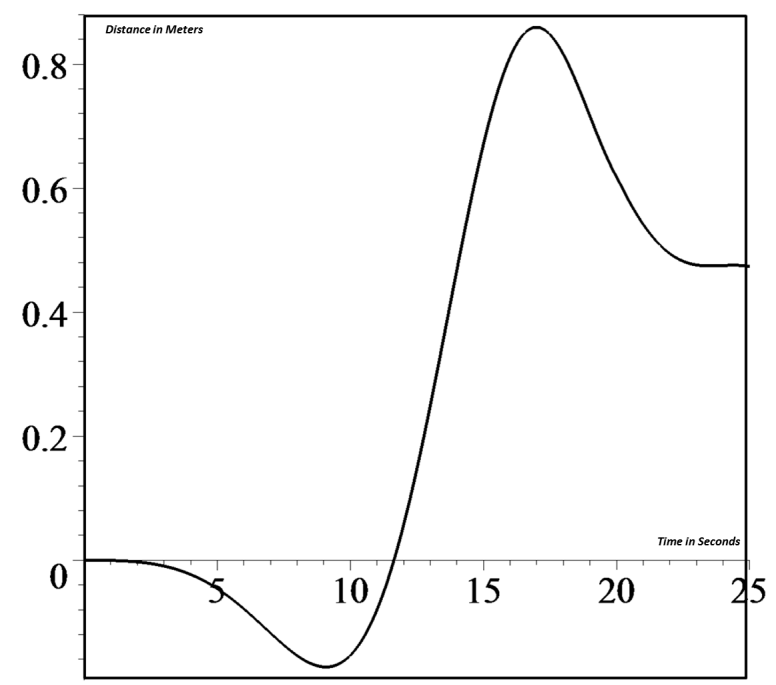

The only problem here is that there is no way to make sure at what time during the motion we are getting the biggest deviation of the two paths. In order to get a more accurate result on the deviation of the two paths, we will have to find a second method for comparing. By comparing only the global centre of rotation of the two paths of motion we can compare the two paths only by their geometry, which is a more accurate way because, firstly what is important for the research is to find the difference in the geometry of the two generated paths and secondly we can make sure this is the biggest deviating distance between the two paths of motion, geometrically.

Doing the above mentioned we will have only $0.19 \mathrm{~m}$ deviation in the $x$ direction and $0.32 \mathrm{~m}$ in the $y$ direction. The total distance of the two global centres of rotation is only $0.37 \mathrm{~m}$ which is just $0.9 \%$ of the radius of rotation.

To conclude, in the case of moving with a constant steering angle but a varying velocity, the resulted path of motion using the steady-state responses of the vehicle or solving the dynamic equations of the motion of the vehicle are definitely close enough to make us able to neglect the difference. So, it is suggested to at least in the case of complicated calculation of equations of motion to use the steady-state responses for solving the problem.

Figure 11 shows the comparison between the two resulted paths of motion and Figure 12 shows the comparison between the movement of the centre of rotation of the two vehicles in their respective body frames. As mentioned earlier the movement of the two vehicles look the same for the passengers, that is why the two lines in Figure 12 are not recognisable. 
Figure 11 The exact and approximate paths of motion (see online version for colours)

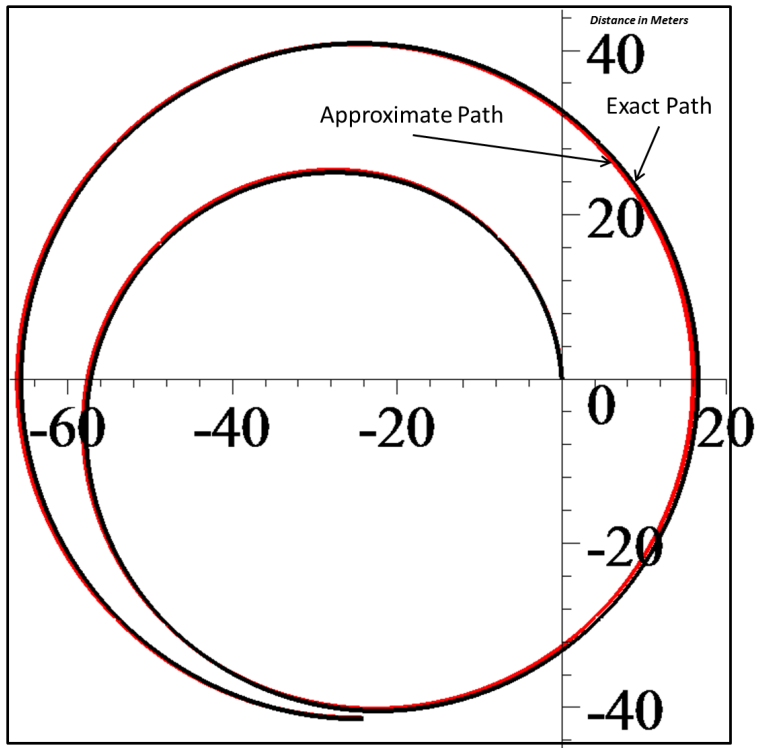

Figure 12 The exact and approximate centres of rotation in the body frame (see online version for colours)

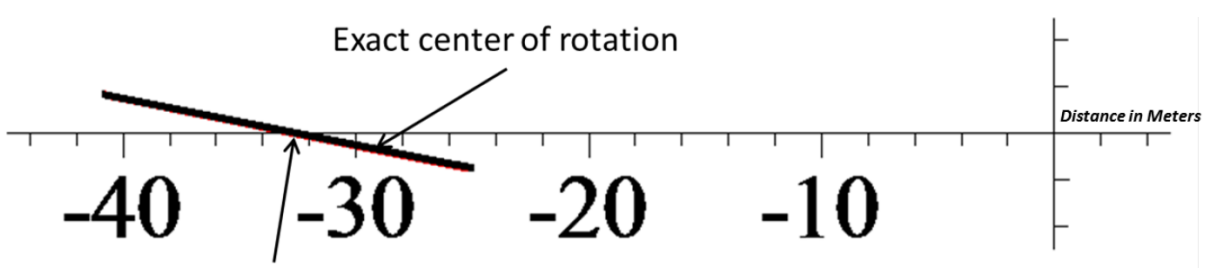

\section{References}

Jazar, R.N. (2010) 'Mathematical theory of autodriver for autonomous vehicles', Journal of Vibration and Control, Vol. 16, No. 2, pp.253-279.

Jazar, R.N. (2011) Advanced Dynamics: Rigid Body, Multibody, and Aerospace Application, John Wiley \& Sons, New York.

Jazar, R.N. (2013) Vehicle Dynamics: Theory and Applications, 2nd ed., Springer, New York.

Tahami, F., Kazemi, R. and Farhanghi, S. (2003) 'A novel driver assist stability system for all-wheel-drive electric vehicles', IEEE Transactions on Vehicular Technology, Vol. 52, No. 3, pp.683-692. 


\section{Nomenclature}

$a \equiv \ddot{x} \quad$ acceleration

$a_{i} \quad$ distance of the axle number $i$ from the mass centre

$[A] \quad$ force coefficient matrix

$B(C x y z) \quad$ vehicle coordinate frame

$C \quad$ mass centre

$C_{\alpha} \quad$ tire sideslip coefficient

$C_{\alpha f} \quad$ front sideslip coefficient

$C_{\alpha r} \quad$ rear sideslip coefficient

$C_{r}, \cdots, D_{\delta}$ force system coefficients

$C_{r} \quad$ proportionality coefficient between $F_{y}$ and $r$

$C_{\beta} \quad$ proportionality coefficient between $F_{y}$ and $\beta$

$C_{\delta} \quad$ proportionality coefficient between $F_{y}$ and $\delta$

$D_{r} \quad$ proportionality coefficient between $M_{z}$ and $r$

$D_{\beta} \quad$ proportionality coefficient between $M_{z}$ and $\beta$

$D_{\delta} \quad$ proportionality coefficient between $M_{z}$ and $\delta$

d frame position vector

$F_{x} \quad$ longitudinal force, forward force, traction force

$F_{y} \quad$ lateral force

$F_{y f} \quad$ front lateral force

$F_{y r} \quad$ rear lateral force

g, $g \quad$ gravitational acceleration

$G(O X Y Z)$ global coordinate frame

I mass moment

K stability factor

$m \quad$ mass

$M_{x} \quad$ roll moment, bank moment, tilting torque

$M_{y} \quad$ pitch moment

$M_{z} \quad$ yaw moment, aligning moment

$p=\dot{\varphi} \quad$ roll rate

q control variable vector 


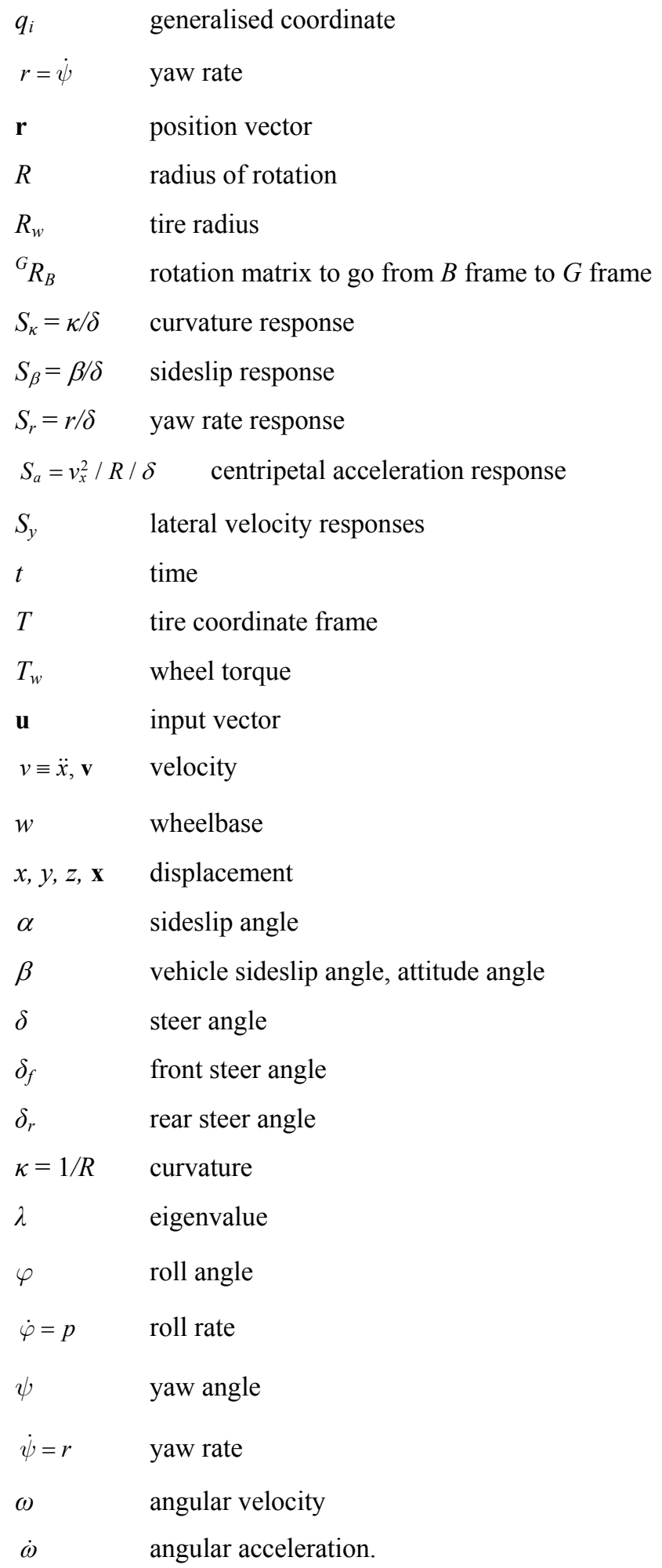

